\title{
Radiocontrast-Induced Acute Kidney Injury Following Coronary Angiography and Percutaneous Coronary Intervention: A Retrospective Cohort Study
}

\author{
Van Bui Pham ${ }^{1, *}$, Quang Dung Nguyen ${ }^{2}$, Nghia Huynh Thi Nguyen ${ }^{3}$, \\ Thanh Phuong Pham Nguyen ${ }^{4}$, Thanh Liem Vo ${ }^{5}$, Minh Cuong Duong ${ }^{6}$ \\ ${ }^{1}$ Department of Nephrology-Urology-Transplantation, Pham Ngoc Thach University of Medicine, Ho Chi Minh City, Vietnam \\ ${ }^{2}$ Department of Cardiology and Cardiovascular Intervention, Nguyen Tri Phuong Hospital, Ho Chi Minh City, Vietnam \\ ${ }^{3}$ Department of Internal Medicine, Pham Ngoc Thach University of Medicine, Ho Chi Minh City, Vietnam \\ ${ }^{4}$ Department of Biostatistics, Epidemiology and Informatics, Perelman School of Medicine, University of Pennsylvania, Philadelphia, USA \\ ${ }^{5}$ Department of Family Medicine, Pham Ngoc Thach University of Medicine, Ho Chi Minh City, Vietnam \\ ${ }^{6}$ School of Public Health and Community Medicine, University of New South Wales, Kensington, Australia
}

\section{Email address:}

phamvanbui@pnt.edu.vn (V. B. Pham), bui.pham@fvhospital.com (V. B. Pham), buimy55@yahoo.com (V. B. Pham), quangdungdr@gmail.com (Q. D. Nguyen), nghiahuynh1204@yahoo.com (N. H. Thi Nguyen),

tphuong.phamnguyen@gmail.com (T.P.P. Nguyen), thanhliem.vo@gmail.com (T. L. Vo), minh.duong@unsw.edu.au (M. C. Duong)

${ }^{*}$ Corresponding author

\section{To cite this article:}

Van Bui Pham, Quang Dung Nguyen, Nghia Huynh Thi Nguyen, Thanh Phuong Pham Nguyen, Thanh Liem Vo, Minh Cuong Duong. Radiocontrast-Induced Acute Kidney Injury Following Coronary Angiography and Percutaneous Coronary Intervention: A Retrospective Cohort Study. American Journal of Internal Medicine. Vol. 6, No. 5, 2018, pp. 138-143. doi: 10.11648/j.ajim.20180605.18

Received: August 22, 2018; Accepted: September 10, 2018; Published: October 10, 2018

\begin{abstract}
Despite the remarkable technological advances in coronary angiography $(\mathrm{CA}) /$ percutaneous coronary intervention (PCI) for diagnosis and treatment of coronary artery disease, the contrast-induced acute kidney injury (CI-AKI) is always an important cause of hospital-acquired AKI. Most local CI-AKI studies in Vietnam had small sample sizes and short-term follow-up of only 24-48 hours following CA or PCI intervention, resulting in controversial conclusions. We conducted a study of the incidence of CI-AKI during a longer follow-up time period and associated risk factors among adult patients undergoing CA/ PCI at Nguyen Tri Phuong University Public Hospital and Tam Duc Private Cardiology Center in Ho Chi Minh City, Vietnam between January 2014 and March 2015. All 320 patients with CA/PCI at the two hospitals were enrolled in a retrospective cohort study. Information on demographic data, treatment, and laboratory test results was collected from the patients' records. The total cumulative incidence of CI-AKI at 24, 48, 72 and $\geq 72$ hours following CA/PCI was $6.7 \%, 12 \%$, $14 \%$ and $16.9 \%$ respectively. Prognostic factors for CI-AKI included an increase by $1 \mathrm{ml} / \mathrm{min} / 1,73 \mathrm{~m}^{2}$ in clearance creatinine before the intervention $(\mathrm{P}=0.006$, Hazard Ratio $(\mathrm{HR})=0.970,95 \% \mathrm{CI} 0.949-0.991)$ and an increase by $1 \%$ in ejection fraction $(\mathrm{P}=0.023, \mathrm{HR}=0.984,95 \% \mathrm{CI} 0.970-0.998)$. Delayed CI-AKI was not rare after CA/PCI intervention. Therefore, it is pivotal to monitor serum creatinine in a longer time after the intervention to timely detect CI-AKI. Also, information on risk factors such as emergency interventions, chronic kidney disease, and ejection fraction $<45 \%$ could assist in predicting CI-AKI development.
\end{abstract}

Keywords: Acute Kidney Injury, Coronary Angiography, Percutaneous Coronary Intervention, Radiocontrast Media

\section{Introduction}

Coronary angiography (CA) has been the gold standard for the diagnosis of coronary artery disease (CAD) which is one of the most common causes of death [1]. Although the newer generations of radiocontrast are well tolerated, rare adverse effects such as acute kidney injury (AKI) remain a persistent problem and can be life-threatening. According to many 
studies, contrast-induced acute kidney injury (CI-AKI) is the third most common cause of hospital-acquired AKI [2-4]. This condition results in a permanent loss of kidney function and an increased mortality rate among in-hospital patients [5, 6]. CI-AKI also increases risks of myocardial infarction (MI), hemorrhage, prolonged hospital length of stay, and dialysis, resulting in increased treatment cost for patients with coronary interventions [7, 8]. Risk factors for CI-AKI include high-dose radiocontrast, chronic kidney disease (CKD), diabetes mellitus (DM), old age, severe heart failure (HF), peri-interventional cardiovascular instability, coadministration of nephrotoxic drugs, dyslipidemia, hypertension (HT) and anemia [9-12]. A careful assessment and detection of these factors are crucial in preventing CIAKI, and thereby improving the efficacy of coronary intervention and patient's outcome.

In Vietnam, coronary intervention has become more widely performed, and the risk of CI-AKI would therefore increase in parallel [13]. However, published studies on this complication are scarce. Most studies were single center and focused on creatinine changes at or less than 48 hours. The presenting study was conducted at the two first-rank general and cardiology hospitals in Ho Chi Minh City, Vietnam, with the aim to assess the incidence and associated risk factors of CI-AKI by long-term monitoring serum creatinine.

\section{Materials and Methods}

\subsection{Design of the Study}

A retrospective cohort study was conducted at Nguyen Tri Phuong (NTP) University Public Hospital and Tam Duc (TD) Private Cardiology Center between January 2014 and March 2015. All patients aged 18 years and older with CA or percutaneous coronary intervention (PCI) were recruited to participate in the study. The study protocol was approved by the ethics committee/ Board of Directors of NTP and TD hospitals.

We proposed the hypothesis that the cumulative incidence of CI-AKI events would increase while the serum creatinine was being monitored with time. Therefore, the objective of our study was also to evaluate the moments for the occurrence of CI-AKI event. As the events would change with time, and each individual had variable follow-ups, we used the exponential model to calculate the minimal event number for survival regression analysis. Assuming that the variables of the patients in two hospitals were similar despite the difference in the number of patients in each hospital, the number of event occurring at each hospital would be calculated by the following equation [14]

$$
\mathrm{n}>\frac{2 *\left(\mathrm{Z}_{1-\frac{\alpha}{2}}+\mathrm{Z}_{1-\beta}\right)^{2}}{\ln (\theta)^{2}}
$$

In which $\ln (\theta)$ was considered as relative risk (RR). According to the literature, the RR of CI-AKI in patients with coronary intervention (CI) would be three times higher than that of patients without CI [15]. Z1- $\alpha / 2$ was conventionally set at 1.96 , corresponding to a statistic threshold of $\alpha=0.05$. Z1- $\beta$ was considered as the strength of a statistic test. We expected $\beta=0.1$, and thus $Z 1-\beta$ value was 1.28. Based on these factors, the minimal event rate would be $\mathrm{n}>17.4$, and thus the total events of the two hospitals would be 35. According to the literature, CI-AKI incidence ranged from $7-15 \%$ [16]. If $15 \%$ was taken as CI-AKI incidence, the total sample size of our study would be at least 233 patients.

Patients' records were utilized to collect information on demographic data (age and sex), medical information and laboratory test results. Medical information included symptoms at admission, pre-intervention ejection fraction (EF) measured by echocardiography, medical history and comorbidities, intervention types (CA, elective or emergency PCI procedures), types of radiocontrast used, and length of hospital stay. Laboratory test results included serum creatinine before intervention, at 24 hours, 48 hours, 72 hours, and $>72$ hours following intervention and blood biochemistries at admission (hemoglobin, glucose, HBA1C, total cholesterol, high density lipoprotein (HDL), low density lipoprotein (LDL) cholesterol, and triglycerides). All laboratory tests were performed at study clinics' validated and qualified laboratories that meet the national laboratory standard. Pre-intervention clearance creatinine $(\mathrm{CrCl})$ was estimated by using Cockcroft-Gault formula [17, 18]. Cigarroa score which is the maximum acceptable contrast dose and is defined as $(5 \mathrm{~mL} \times$ body weight $(\mathrm{kg})) /$ baseline serum creatinine $(\mathrm{mg} / \mathrm{dL})$ ) was calculated for comparison [19]. The recommended Cigarroa score is $\leq 5$ [19]. CI-AKI was defined as a condition in which the serum creatinine following the intervention increases up to $25 \%$ higher than the value before intervention [16].

\subsection{Statistical Analysis}

Data were managed and analyzed using Statistical Package for the Social Sciences (SPSS) version 20 (IBM). Categorical variables were expressed as a number and percentage, while continuous variables were presented by mean and standard deviation (SD). Cox multiple-regression analysis was used to test risk factors for CI-AKI. Independent variables which were used for the model included demographics variables, reasons for admission, medical history, serum biochemistries at admission, pre-intervention $\mathrm{CrCI}$ and $\mathrm{EF}$, intervention types, types of radiocontrast used, and hospital that provided treatment (e.g. NTP and TD). Statistic significant level was set at $\mathrm{P} \leq 0.05$.

\section{Results}

\subsection{Characteristics of Study Population}

A total of 320 patients participated in the study of whom 112 were from NTP and 208 from TD (Table 1). The mean age (year) of all patients was $64.9 \pm 12.6$ and $65.9 \%$ were male. The most common reason for admission was angina pectoris $(231 / 320,72 \%)$ and nearly three fourth $(235 / 320$, 
$73.5 \%$ ) of study participants had hypertension. The mean length of hospital stay was $10.3 \pm 7.6$ days. The intervention types in order of frequency included elective percutaneous coronary intervention (159/320, 49.7\%), emergency percutaneous coronary intervention $(110 / 320,34.4 \%)$ and coronary angiography only $(51 / 320,15.9 \%)$. The radiocontrasts used were Ominpaque, Ultravist, Xenetix, and
Visipaque. Xenetix (170/320, 53.1\%) was most commonly prescribed in both hospitals while Visipaque was only used in TD. There were 22 patients $(6.9 \%)$ who received the contrast dose larger than the recommendation (e.g. Cigarroa score $>5)$. There were two CI-AKI cases treated by temporary hemodialysis (data not shown).

Table 1. Demographic and clinical characteristics of 320 patients receiving treatment at Nguyen Tri Phuong Hospital and Tam Duc Cardiology Center.

\begin{tabular}{|c|c|c|c|}
\hline Characteristics & $\operatorname{NTP}(N=112)$ & TD $(\mathrm{N}=208)$ & Both hospitals $(\mathrm{N}=320)$ \\
\hline \multicolumn{4}{|l|}{ Demographic characteristics } \\
\hline Male n (\%) & $72(64.3)$ & $139(66.8)$ & $211(65.9)$ \\
\hline Age (year) mean (SD) & $63.0(12.2)$ & $65.9(12.7)$ & $64.9(12.6)$ \\
\hline \multicolumn{4}{|l|}{ Parameters at admission $\mathrm{n}(\%)$} \\
\hline Anemia & $42(39.3)$ & $83(39.9)$ & $125(39.7)$ \\
\hline Ejection fraction $\leq 40 \%$ & $13(12.6)$ & $46(22.1)$ & $59(19.0)$ \\
\hline Dyspnea & $40(35.7)$ & $49(23.6)$ & $89(27.8)$ \\
\hline Angina & $85(75.9)$ & 99 (47.6) & $184(57.5)$ \\
\hline \multicolumn{4}{|l|}{ Blood biochemistries at admission mean (SD) } \\
\hline Hemoglobin $(\mathrm{g} / \mathrm{dl})$ & $13.2(1.9)$ & $13(1.8)$ & $13.1(1.8)$ \\
\hline Serum creatinine $(\mathrm{mmol} / \mathrm{l})$ & $108.3(52.6)$ & $108.4(90.3)$ & $108.4(79.2)$ \\
\hline Serum glucose (mmol/l) & $8.2(3.9)$ & $6.6(2.1)$ & $7.1(2.9)$ \\
\hline $\operatorname{HbA1C}(\%)$ & $6.2(1.9)$ & $6.1(1.7)$ & $6.2(1.7)$ \\
\hline Uric Acid $(\mu \mathrm{mol} / \mathrm{L})$ & $415(198.7)$ & $365.4(127.4)$ & $366.8(128.8)$ \\
\hline Cholesterol (mmol/L) & $4.9(1.1)$ & $4.2(1.5)$ & $4.4(1.5)$ \\
\hline $\mathrm{LDL}(\mathrm{mmol} / \mathrm{L})$ & $2.6(1.1)$ & $3.1(1.8)$ & $2.9(1.6)$ \\
\hline $\mathrm{TG}(\mathrm{mmol} / \mathrm{L})$ & $2.7(1.5)$ & $3.0(1.8)$ & $2.9(1.7)$ \\
\hline $\mathrm{HDL}(\mathrm{mmol} / \mathrm{L})$ & $1.0(0.3)$ & $1.1(0.6)$ & $1.1(0,5)$ \\
\hline \multicolumn{4}{|l|}{ Medical history n (\%) } \\
\hline Hypertension & $83(74.1)$ & $152(73.1)$ & $235(73.4)$ \\
\hline Diabetes mellitus & $31(27.7)$ & $74(35.6)$ & $105(32.8)$ \\
\hline Myocardial infarction & $28(25)$ & $57(27.4)$ & $85(26.6)$ \\
\hline Heart Failure & $12(10.7)$ & $17(8.2)$ & $29(9.1)$ \\
\hline Coronary artery bypass & $1(0.9)$ & $8(3.8)$ & $9(2.8)$ \\
\hline Stroke & $11(9.8)$ & $4(1.9)$ & $15(4.7)$ \\
\hline Peripheral Artery Disease & $2(1.8)$ & $6(2.9)$ & $8(2.5)$ \\
\hline Chronic Kidney Disease & $4(3.6)$ & $30(14.4)$ & $34(10.6)$ \\
\hline Smoking & $48(42.9)$ & $93(44.7)$ & $141(44.1)$ \\
\hline \multicolumn{4}{|l|}{ Radiocontrasts used n (\%) } \\
\hline Ominpaque & $1(0.9)$ & $52(25)$ & $53(16.6)$ \\
\hline Ultravist & $5(4.5)$ & $0(0)$ & $5(1.6)$ \\
\hline Visipaque & $0(0)$ & $92(44.2)$ & $92(28.8)$ \\
\hline Xenetix & $106(94.6)$ & $64(30.8)$ & $170(53.1)$ \\
\hline \multicolumn{4}{|l|}{ Intervention Type n (\%) } \\
\hline Cigarroa score $>5$ & $13(19.1)$ & $9(4.7)$ & $22(6.9)$ \\
\hline Coronary angiography only & $21(18.8)$ & $30(14.4)$ & $51(15.9)$ \\
\hline Elective percutaneous coronary intervention & $45(40.2)$ & $114(54.8)$ & $159(49.7)$ \\
\hline Emergency percutaneous coronary intervention & $46(41.0)$ & $64(30.8)$ & $110(34.4)$ \\
\hline \multicolumn{4}{|l|}{ Other characteristic mean (SD) } \\
\hline Length of hospital stay (days) & $9.6(6.1)$ & $10.6(8.3)$ & $10.3(7.6)$ \\
\hline
\end{tabular}

\subsection{Incidence of Contrast-induced Acute Kidney Injury}

Serum creatinine level was measured at 24 hours, 48 hours, 72 hours and $>72$ hours after the intervention. At 24 hours, nearly all $(313 / 320,97.8 \%)$ study participants were undertaken serum creatinine measurement (Table 2). The total proportion of patients whose serum creatinine level was measure decreased to $52.2 \%$ (167/320), 46.9\% (150/320), and $25.9 \%(83 / 320)$ at 48 hours, 72 hours and $>72$ hours respectively. At 24 hours and 48 hours after the intervention, the total incidence of CI-AKI was $6.7 \%(21 / 313)$ and $12 \%$ (20/167) respectively (Table 2$)$. The incidence of CI-AKI at NTP was significantly higher than the incidence of CI-AKI at TD at 24 hours $(\mathrm{P}=0.002)$ and 48 hours $(\mathrm{P}=0.001)$. At 72 hours and $>72$ hours after the intervention, the total incidence of CI-AKI was $14 \%(21 / 150)$ and $16.9 \%$ (14/83) respectively and there was no difference in the incidence of CI-AKI between the two hospitals ( $\mathrm{P}>0.05)$. 
Table 2. Incidence of contrast-induced acute kidney injury (CI-AKI) at different time points after the intervention at Nguyen Tri Phuong Hospital (NTP) and Tam Duc (TD) Cardiology Center.

\begin{tabular}{|c|c|c|c|c|c|c|c|c|}
\hline \multirow[b]{2}{*}{ Time points } & \multicolumn{3}{|c|}{$\begin{array}{l}\text { Number of patients whose serum creatinine } \\
\text { was measured }\end{array}$} & \multicolumn{3}{|c|}{$\begin{array}{l}\text { Incidence of contrast-induced acute kidney } \\
\text { injury (CI-AKI) }\end{array}$} & \multirow{2}{*}{$\begin{array}{l}P^{\&} \\
\text { value }\end{array}$} & \multirow[b]{2}{*}{ OR (95\%CI) } \\
\hline & $\begin{array}{l}\text { Both hospitals } \\
(\mathbf{N}=320) n \\
(\%)\end{array}$ & $\begin{array}{l}\operatorname{NTP}(N= \\
112) \\
n(\%)\end{array}$ & $\begin{array}{l}\text { TD(N }=208) \\
\text { n }(\%)\end{array}$ & $\begin{array}{l}\text { Both } \\
\text { hospitals* } \\
\text { n (\%) }\end{array}$ & NTP*n (\%) & TD*n (\%) & & \\
\hline At 24 hours & $313(97.8)$ & $109(97.3)$ & $204(98.1)$ & $21(6.7)$ & $14(12.8)$ & $7(3.4)$ & 0.002 & $0,24(0,09-0,62)$ \\
\hline At 48 hours & $167(52.2)$ & $31(27.7)$ & $136(65.4)$ & $20(12.0)$ & $9(29.0)$ & $11(8.1)$ & 0.001 & $0,22(0,08-0,58)$ \\
\hline At 72 hours & $150(46.9)$ & $44(39.3)$ & $106(51.0)$ & $21(14.0)$ & $6(13.6)$ & $15(14.2)$ & 0.934 & $1,04(0,38-2,89)$ \\
\hline At $>72$ hours & $83(25.9)$ & $16(14.3)$ & $67(32.2)$ & $14(16.9)$ & $3(18.8)$ & $11(16.4)$ & 0.823 & $0,85(0,21-3,49)$ \\
\hline
\end{tabular}

*Patients whose serum creatinine level meets the definition of CI-AKI at the given follow-up were counted

${ }^{\&}$ Chi-squared test

At 24 hours after the intervention, the number of CI-AKI incident cases at NTP and TD was 14 and 7 respectively (Table 3). The cumulative frequency of CI-AKI at NTP increased to 18 at 48 hours and remained the same at 72 hours and $>72$ hours. In contrast, cumulative frequency of CI-AKI at NTP increased by time which was 14 (at 48 hours), 22 (at 72 hours) and 27 (at $>72$ hours). Among 21 patients undergoing coronary angiography only for diagnosis, $12 \%$ developed CI-AKI (data not shown). There was $10.3 \%$ of a total 45 patients receiving elective interventions had CIAKI, while $21.3 \%$ of 46 patients undergoing emergency interventions developed CI-AKI (data not shown).

Table 3. Cumulative frequency of contrast-induced acute kidney injury (CI-AKI) at different time points after the intervention at Nguyen Tri Phuong Hospital (NTP) and Tam Duc (TD) Cardiology Center.

\begin{tabular}{llll}
\hline \multirow{2}{*}{ Time points } & Cumulative frequency of CI-AKI & & \\
\cline { 2 - 4 } & Both hospitals* & NTP* & TD* \\
\hline At 24 hours & 21 & 14 & 7 \\
At 48 hours & 32 & 18 & 14 \\
At 72 hours & 40 & 18 & 22 \\
At $>72$ hours & 45 & 18 & 27 \\
\hline
\end{tabular}

*Only new patients whose serum creatinine level meets the definition of CI-AKI at the given follow-up were cumulatively counted

\subsection{Risk Factors for Contrast-induced Acute Kidney Injury}

Cox multiple-regression analysis indicated that having an increase by $1 \mathrm{ml} / \mathrm{min} / 1.73 \mathrm{~m}^{2}$ in pre-intervention $\mathrm{CrCl}$ $(\mathrm{P}=0.006, \mathrm{HR}=0.970)$ and an increase by $1 \%$ in preintervention $\mathrm{EF} \quad(\mathrm{P}=0.023, \mathrm{HR}=0.984)$ were independent prognostic factors for CI-AKI (Table 4). Other demographic variables, symptoms at admission, medical history, blood biochemistries at admission, hospital that provided treatment (e.g. NTP and TD), and types of radiocontrast used were not significantly associated with $\mathrm{CI}-\mathrm{AKI}$ ( $\mathrm{P}>0.05$, data not shown).

Table 4. Cox multiple-regression analysis for risk factors for contrast-induced acute kidney injury (CI-AKI) in patients receiving interventions at Nguyen Tri Phuong Hospital (NTP) and Tam Duc (TD) Cardiology Center.

\begin{tabular}{|c|c|c|c|}
\hline Characteristics & Beta Coefficient & P value & HR $(95 \% C I)$ \\
\hline Pre-intervention clearance creatinine increased by $1\left(\mathrm{ml} / \mathrm{min} / 1.73 \mathrm{~m}^{2}\right)$ & -0.031 & 0.006 & $0.970(0.949-0.991)$ \\
\hline Pre-intervention ejection fraction increased by $1 \%$ & -0.016 & 0.023 & $0.984(0.970-0.998)$ \\
\hline
\end{tabular}

\section{Discussion}

CI-AKI incidence varies depending on the diagnosis criteria. The widely accepted definition of CI-AKI is the $25 \%$ increase in serum creatinine level at 48-72 hours after intervention compared to baseline (serum creatinine level before intervention) $[16,20,21]$. Based on this definition, we found that the total cumulative incidence of CI-AKI at 24, 48,72 and $>72$ hours following CA/PCI was $6.7 \%, 12 \%$, $14 \%$, and $16.9 \%$, respectively among our study participants whose serum creatinine was measured at these time points. It is documented that in some patients undergoing $\mathrm{CA} / \mathrm{PCI}$ intervention serum creatinine only temporarily increases then immediately returns to baseline [22, 23]. If serum creatinine value was only documented at a specific moment, we could inadvertently omit many CI-AKI cases, resulting in a falsely low incidence. In our study, serum creatinine level was monitored during a longer period of time (up to 72 hours or more after intervention). This enabled us to reveal some delayed CI-AKI events, which could not be detected in the common follow-up period of less than 48 hours. Also, CIAKI events were calculated by using Cox survival regression model with cumulative incidence with time. This helped to reflect better the incidence of CI-AKI in reality and therefore explain why CI-AKI incidence in our study was higher than other studies in Vietnam which utilized a shorter follow-up [24, 25].

Of various interventions, the risk of developing CI-AKI among patients undergoing emergency percutaneous coronary intervention was reported to be significantly higher than in those undergoing elective intervention [26]. This 
demonstrated the importance of patient preparations, while considering the importance of hemodynamic instability management in preventing CI-AKI, which was also reported in other study [16]. In a recent study including 2,025 patients with ST-segment-elevation myocardial infarction managed by primary PCI, Caspi $\mathrm{O}$ et al. found that CI-AKI development following primary PCI was not related to the use of contrast media, but rather to factors such as older age ( $\geq 70$ years) and hemodynamic instability [27]. Our findings are in line with this study regarding the types of contrast media used. Yet we found no association between CI-AKI and demographic characteristics.

We found that having CKD was an independent risk factor for CI-AKI. There was a statistically significant relationship between the decrease in $\mathrm{CrCl}$ and CI-AKI. In fact, a decrease in pre-intervention $\mathrm{CrCI}$ by $1\left(\mathrm{ml} / \mathrm{min} / 1.73 \mathrm{~m}^{2}\right)$ increased the risk of CI-AKI by 1.03 times. This finding was in consistence with other studies [7, 27].

Heart failure is a complex clinical syndrome with diastolic and/or systolic dysfunction secondary to structural or functional injury of the heart, clinically manifested by dyspnea, volume retention, and exhaustion [28]. Several studies revealed that an $\mathrm{EF} \leq 40 \%$ was an independent risk factor for CI-AKI [11, 27]. Indeed, compared with those with Killip class I, patients with Killip class II-III had 1.99 fold greater odds of acquiring CI-AKI [27]. Patients with Killip class IV or who used an intra-aortic balloon pump had a 7.43-fold higher odds of acquiring CI-AKI. Patients with an EF below $45 \%$ had a 1.44-fold higher odds of acquiring CI-AKI [27]. Our study found that an increase in $1 \%$ of EF would decrease the risk of acquiring CI-AKI by 0.984 times.

Current guidelines [10,29] recommend using low osmolar or iso-osmolar radiocontrast instead of high osmolar radiocontrast in cardiovascular interventions to minimize the risk of CI-AKI. However, there was no difference in the rate of CI-AKI found among four kinds of radiocontrast used in our study. In addition, like the other study [27], we did not find any relationship between doses of radiocontrast and CIAKI.

The small number of patients whose serum creatinine was documented during different time points is a notable limitation of our study due to the common local practice of early discharges of some patients. This may possibly underestimate the incidence of CI-AKI after CA/PCI intervention. Yet we successfully recruited all study participants receiving treatment at both the largest public hospital and private center for cardiovascular intervention in southern Vietnam to demonstrate the pattern of CI-AKI following $\mathrm{CA} / \mathrm{PCI}$ intervention and associated risk factors in these different health clinics.

\section{Conclusions}

Delayed CI-AKI following CA/PCI intervention was not uncommon but not systematically detected. Our findings emphasized the importance of serum creatinine monitoring during a longer period after CA/PCI to timely detect CI$\mathrm{AKI}$; the longer post-interventional period in which the patients' serum creatinine levels were closely monitored, the CI-AKI incidence was higher. Also, information on risk factors such as emergency interventions (without patient careful preparation), chronic kidney disease, and ejection fraction $<45 \%$ could assist in predicting CI-AKI development. In addition, there was no difference in the rate of CI-AKI found among four kinds of radiocontrast used in our study nor the relationship between doses of radiocontrast and $\mathrm{CI}-\mathrm{AKI}$.

\section{Conflicts of Interest}

All the authors do not have any possible conflicts of interest.

\section{References}

[1] Kizilbbash Mohammad, Parker Jeffrey R., Sarwar Muhammad A., et al. Ischemic Heart Disease. In: The washington manual medical therapeutics. 34 ed: Lippincott Williams \& Wilkins; 2014:112-70.

[2] Seeliger E., Sendeski M., Rihal C. S., et al. Contrast-induced kidney injury: mechanisms, risk factors, and prevention. European heart journal. [2012];33:2007-15.

[3] Finn W. F. The clinical and renal consequences of contrastinduced nephropathy. Nephrol Dial Transplant. [2006];21:i210 .

[4] Rear R., Bell R. M., Hausenloy D. J. Contrast-induced nephropathy following angiography and cardiac interventions. Heart. [2016];102:638-48.

[5] Rudnick MR. Pathogenesis, clinical features, and diagnosis of contrast-induced nephropathy. In: Palevsky PM, Sheridan AM, eds. UpToDate. Alphen aan den Rijn, Netherlands: UpToDate; 2017.

[6] Maioli M., Toso A., Leoncini M., et al. Persistent renal damage after contrast-induced acute kidney injury: incidence, evolution, risk factors, and prognosis. Circulation. [2012];125:3099-107.

[7] Solomon R. Contrast-induced acute kidney injury: is there a risk after intravenous contrast? Clinical journal of the American Society of Nephrology : CJASN. [2008];3:1242-3.

[8] Aubry P., Brillet G., Catella L., et al. Outcomes, risk factors and health burden of contrast-induced acute kidney injury: an observational study of one million hospitalizations with image-guided cardiovascular procedures. BMC nephrology. [2016];17:167.

[9] Xu J., Zhang M., Ni Y., et al. Impact of low hemoglobin on the development of contrast-induced nephropathy: A retrospective cohort study. Experimental and therapeutic medicine. [2016];12:603-10.

[10] Disease Kidney. Improving Global Outcomes (KDIGO) Acute Kidney Injury Work Group. KDIGO Clinical Practice Guideline for Acute Kidney Injury. Kidney inter., Suppl. [2012];2:1-138. 
[11] Mehran R., Aymong E. D., Nikolsky E., et al. A simple risk score for prediction of contrast-induced nephropathy after percutaneous coronary intervention: development and initial validation. Journal of the American College of Cardiology. [2004];44:1393-9.

[12] Li W. H., Li D. Y., Han F., et al. Impact of anemia on contrastinduced nephropathy (CIN) in patients undergoing percutaneous coronary interventions. International urology and nephrology. [2013];45:1065-70.

[13] Suc Khoe \& Doi Song. Vietnam has more than 50 coronary interventional centers [in Vietnamese]. 2015; http://www.yhth.vn/viet-nam-da-co-tren-50-don-vi-tim-machcan-thiep_d3338.aspx. Accessed 2 May, 2018.

[14] Weaver MA. Sample Size Calculations for Survival Analysis. 2009;

http://www.icssc.org/Documents/AdvBiosGoa/Tab\%2026.00 SurvSS.pdf. Accessed 2 April, 2017.

[15] Ozkok S., Ozkok A. Contrast-induced acute kidney injury: A review of practical points. World journal of nephrology. [2017];6:86-99.

[16] McCullough P. A. Contrast-induced acute kidney injury. Journal of the American College of Cardiology. [2008];51:1419-28.

[17] Bui Van Pham. Manual of the Diagnosis and the Treatment of Common Diseases in Internal Medicine [in Vietnamese]. In: Chronic Kidney Disease. Vietnam: Medicine Publishing; 2008:97-128.

[18] DW Cockcroft, MH Gault. Prediction of creatinine clearance from serum creatinine. . Nephron. [1976];16:31-41.

[19] Cigarroa R. G., Lange R. A., Williams R. H., et al. Dosing of contrast material to prevent contrast nephropathy in patients with renal disease. Am J Med. [1989];86:649-52.

[20] Bouzas-Mosquera Alberto. Contrast-induced nephropathy and acute renal failure following urgent cardiac catheterization: incidence, risk factor, and prognosis. Revista espanola de cardiologia. [2007];60:1026-34.

[21] Neyra J. A., Shah S., Mooney R., et al. Contrast-induced acute kidney injury following coronary angiography: a cohort study of hospitalized patients with or without chronic kidney disease. Nephrol Dial Transplant. [2013];28:1463-71.

[22] Wi J., Ko Y. G., Kim J. S., et al. Impact of contrast-induced acute kidney injury with transient or persistent renal dysfunction on long-term outcomes of patients with acute myocardial infarction undergoing percutaneous coronary intervention. Heart. [2011];97:1753-7.

[23] Basu A. Contrast-induced nephropathy. 2017; https://emedicine.medscape.com/article/246751-overview. Accessed 11 March, 2018

[24] Minh Thien Doan Nguyen, Nhan Thanh Vo. Serum cystatin in diagnosis of contrast-induced nephropathy early after percutaneos coronary intervention [in Vietnamese]. Ho Chi Minh City Journal of Medicine. 2010;14(2):93-100.

[25] Nguyen DC, Ho TD, Chau VV. The effect of contrast medium on renal function in patients with coronary intervention [in Vietnamese]. Ho Chi Minh City Journal of Medicine. 2012;16(1):83-87.

[26] Giacoppo et al. Impact of Contrast-Induced Acute Kidney Injury After Percutaneous Coronary Intervention on Shortand Long-Term Outcomes: Pooled Analysis From the HORIZONS-AMI and ACUITY Trials. Circulation Cardiovascular interventions. 2015;8:e002475.

[27] Caspi O., Habib M., Cohen Y., et al. Acute Kidney Injury After Primary Angioplasty: Is Contrast-Induced Nephropathy the Culprit? Journal of the American Heart Association. [2017];6.

[28] Yancy C. W., Jessup M., Bozkurt B., et al. 2013 ACCF/AHA guideline for the management of heart failure: a report of the American College of Cardiology Foundation/American Heart Association Task Force on Practice Guidelines. Journal of the American College of Cardiology. [2013];62:e147-239.

[29] Windecker S, Kolh P, Alfonso F, et al. 2014 ESC/EACTS Guidelines on myocardial revascularization: The Task Force on Myocardial Revascularization of the European Society of Cardiology (ESC) and the European Association for CardioThoracic Surgery (EACTS)Developed with the special contribution of the European Association of Percutaneous Cardiovascular Interventions (EAPCI). European heart journal. 2014;35(37):2541-2619. 